\title{
L-MOMENTS AND MAXIMUM LIKELIHOOD ESTIMATION FOR THE COMPLEMENTARY BETA DISTRIBUTION WITH APPLICATIONS ON TEMPERATURE EXTREMES
}

\author{
Josmar Mazucheli ${ }^{1}$, André Felipe Berdusco Menezes ${ }^{2 *}$ \\ Department of Statistics, Universidade Estadual de Maringá, Maringá, PR, Brasil
}

\begin{abstract}
Although the two-parameter Beta distribution is the standard distribution for analyzing data in the unit interval, there are in the literature some useful and interesting alternatives which are often under-used. An example is the two parameter complementary Beta distribution, introduced by Jones (2002) and, to the best of our knowledge, used only by Iacobellis (2008) as a probabilistic model for the estimation of $T$ year flow duration curves. In his paper the parameters of complementary Beta distribution were successfully estimated, perhaps due to its simplicity, by means of the $L$-moments method. The objective of this paper is to compare, using Monte Carlo simulations, the bias and mean-squared error, of the estimators obtained by the methods of $L$-moments and maximum likelihood. The simulation study showed that the maximum likelihood method has bias and mean -squared error lower than $L$-moments. It is also revealed that the parameters estimated by the maximum likelihood are negatively biased, while by the $L$-moments method the parameters are positively biased. Data on relative indices from annual temperature extremes (percentage of cool nights, percentage of warm nights, percentage of cool days and percentage of warm days) in Uruguay are used for illustrative purposes.
\end{abstract}

Keywords: maximum likelihood estimation, $L$-moments, complementary Beta, Monte Carlo simulation.

\footnotetext{
* Corresponding author:

Email:andrefelipemaringa@gmail.com
} 


\section{Introduction}

It is well known that Beta distribution in its standard form is the most important probability distribution for data analysis with support on the unit interval. Its probability density function (p.d.f.) is very versatile and several uncertainties may be useful modeled by them. A random variable $X$ has a Beta distribution with shape parameters $\alpha$ and $\beta$, both positive, if its p.d.f is expressed as:

$$
f(x \mid \alpha, \beta)=\frac{1}{B(\alpha, \beta)} x^{\alpha-1}(1-x)^{\beta-1}
$$

where $0<x<1$ and $B(\alpha, \beta)=\int_{0}^{1} t^{\alpha}(1-t)^{\beta-1} d t$ is the complete Beta function, also called the Euler integral of the first kind. The corresponding cumulative distribution function (c.d.f.) is the regularized incomplete Beta function and written as:

$$
F(x \mid \alpha, \beta)=\frac{B_{x}(\alpha, \beta)}{B(\alpha, \beta)}
$$

where $B_{x}(\alpha, \beta)$ denotes the incomplete Beta function, i.e $B_{x}(\alpha, \beta)=\int_{0}^{x} t^{\alpha-1}(1-t)^{\beta-1} d t$. For a detailed discussion of Beta distribution interested readers may refer to Johnson et al. (1995), Gupta and Nadarajah (2004) and Nadarajah and Kotz (2007).

Although some distributions also present these characteristics, they are few used in applications. A probability distribution related to Beta the distribution that has not received much attention in the literature was introduced by Jones (2002) and it is obtained by switching the roles of c.d.f. and quantile function of the Beta distribution. Thus, its c.d.f. is given by:

$$
F(x \mid \alpha, \beta)=\oint_{x}(\alpha, \beta)
$$

where $0<x<1$ and $\oint_{x}(\alpha, \beta)$ denotes the inverse of the incomplete Beta ratio, i.e $Q(x \mid \alpha, \beta)=F^{-1}(x \mid \alpha, \beta)$, given in (2). The corresponding p.d.f. is $\frac{d}{d x} Q(x \mid \alpha, \beta)$ and written as follows:

$$
f(x \mid \alpha, \beta)=B(\alpha, \beta) \mid\left[\oint_{x}(\alpha, \beta)\right]^{1-\alpha}\left[1-\oint_{x}(\alpha, \beta)\right]^{1-\beta} .
$$


Jones (2002) named this distribution as complementary Beta distribution (CB), and in the same way as the Beta distribution it is symmetric if and only if $\alpha=\beta$. Also, the parameters $\alpha$ and $\beta$ are symmetrically related by $f(x \mid \alpha, \beta)=f(1-x \mid \beta, \alpha)$, which means that if $X$ has $\mathrm{CB}$ distribution with parameters $\alpha$ and $\beta$ then $1-X$ has a $\mathrm{CB}$ distribution with parameters $\beta$ and $\alpha$. The particular case of (4) for $\alpha=\beta=1$ is the standard Uniform distribution. When $\beta=1$ the $\mathrm{CB}$ distribution reduces to the power function distribution with parameter $1 / \alpha$. For $\alpha=1$ we have the Beta distribution with parameters 1 and $1 / \beta$. In Jones (2007) was showed that CB distribution is a special case, when $a=1-\alpha$ and $b=1-\beta$, of the family of univariate continuous distributions with density function $f(\cdot)$ and distribution function $F(\cdot)$ defined through the relation $f(x)=F^{a}(x)[1-F(x)]^{b}$.

In Figure 1, for selected values of $\alpha$ and $\beta$, the behavior of (1) is displayed can be showed that (1) has the same basic shape properties of the Beta distribution i.e, for $(\alpha, \beta)<$ 1 it is unimodal; $(\alpha, \beta)>1$ it is U-shaped; $(\alpha<1$ and $\beta>1)$ it is J-shaped and for $(\alpha>$ 1 and $\beta<1$ ) reverse $\mathrm{J}$-shaped. These behaviors are quite similar to the Beta distribution with parameters $1 / \alpha$ and $1 / \beta$. Some additional details about $\mathrm{CB}$ distribution can be found in Nadarajah and Kotz (2007).

[Figure 1 about here.]

The CB distribution does not have explicit moments or order $k$ but for $k=1$ and $k=$ 2 the following expressions are provided:

$$
E(X)=\frac{\beta}{\alpha+\beta} \text { and } E\left(X^{2}\right)=\frac{2 B(2 \alpha, 2 \beta+1)}{\alpha[B(\alpha, \beta)]^{2}}{ }_{3} F_{2}(\alpha+\beta, 1,2 \alpha ; \alpha+1,2(\alpha+\beta)+1 ; 1)
$$

where ${ }_{3} F_{2}$ denotes the generalized hypergeometric function (Gradshteyn and Ryzhik, 1994, formula 7.512.5). From Dixon (1902) the definition of $(a, b, c ; d, e ; 1)$ is:

$$
{ }_{3} F_{2}(a, b, c ; d, e ; 1)=\frac{(a / 2) !(a-b) !(a-c) !(a / 2-b-c) !}{a !(a / 2-b) !(a / 2-c) !(a-b-c) !}
$$

where $1+\mathrm{a} / 2-\mathrm{b}-\mathrm{c}$ has a positive real part, $d=a-b+1$ and $e=a-c+1$.

The mean of $\mathrm{CB}$ distribution matches the mean of the Beta distribution with parameters $1 / \alpha$ and $1 / \beta$. In Figure 2 the behaviors of the mean, variance, skewness and kurtosis of the $\mathrm{CB}$ distribution are illustrated as a function of $\alpha$ for some values of $\beta$. It is observed that the skewness may be negative, which implies that $\mathrm{CB}$ distribution might be useful for modeling left-skewed data.

[Figure 2 about here.] 
In spite of $\mathrm{CB}$ distribution has various attractive properties and mostly ones that are complementary to those of the Beta distribution, it has been neglected in the literature. As far as we know, the only real data analysis appeared in Iacobellis (2008). In his paper the CB distribution has been used as a probabilistic model for estimation of $T$ year flow duration curves. Its parameters were successfully estimated by means of $L$-moments.

To the best of our knowledge nothing has been done regarding comparisons among estimators of the $\mathrm{CB}$ distribution. In this paper our main goal is to compare, mainly, by Monte Carlo simulation and real applications, the performance of estimators obtained by the methods of $L$-moments and maximum likelihood. In the same direction, Erişoğlu and Erişoğlu (2014) compared the $L$-moments estimation with maximum likelihood method to estimate the parameters related to mixture of Weibull distributions.

The paper is outline as follows. In Section 2 the $L$-moments and maximum likelihood methods are presented and discussed. The simulations results used to compare these methods, in terms of relative bias and relative root mean-squared error, are presented in Section 3. In Section 4, data on annual temperature extremes in Uruguay are used for illustrative purposes. Some concluding remarks in Section 5 finalize the paper.

\section{Different methods of estimation}

In this section, the estimation of parameters $\alpha$ and $\beta$ of CB distributionis consideredby the $L$-moments and maximum likelihood methods.

\subsection{Method of $L$-moments}

The $L$-moments, whose theory was unified by Hosking (1990), are linear combinations of order statistics and have lower sample variances and are more robust against outliers when compared to the conventional moments. Analogously to the conventional method of moments, the $L$-moments method obtains parameter estimates by equating the first sample $L$-moments to the corresponding theoretical quantities. The theoretical $L$-moments are defined by the quantile function, i.e., the inverse of cumulative distribution function.

Following, for example Hosking and Wallis (1997), the first and second sample $L$-moments are calculated, respectively, from the expressions:

$$
l_{1}=\frac{1}{n} \sum_{i=1}^{n} x_{i: n} \text { and } l_{2}=\frac{2}{n(n-1)} \sum_{i=2}^{n}(i-1) x_{i: n}-l_{1}
$$


where $x_{i: n}$ denotes the $k$ th smallest observation from a sample of size $n$.

For the CB distribution Jones (2002) showed that first and second theoretical $L$-moments are given, respectively, as:

$$
\lambda_{1}(\alpha, \beta)=\frac{\beta}{\alpha+\beta} \text { and } \lambda_{2}(\alpha, \beta)=\frac{\alpha \beta}{(\alpha+\beta)(\alpha+\beta+1)}
$$

Consequently, assuming that $\boldsymbol{x}=\left(x_{1}, \ldots, x_{n}\right)$ is a random sample from (4), the $L$-moments estimates for $\alpha$ and $\beta$, i.e $\hat{\alpha}_{L M E}$ and $\hat{\beta}_{L M E}$ are obtained, respectively, from the following expressions:

$$
\hat{\alpha}_{L M E}=\frac{l_{2}\left(l_{1}-1\right)}{l_{1}^{2}-l_{1}+l_{2}} \text { and } \hat{\beta}_{L M E}=\frac{l_{2} l_{1}}{l_{1}^{2}-l_{1}+l_{2}} .
$$

\subsection{Method of maximum likelihood}

The maximum likelihood method is an alternative to $L$-moments for parameters estimation. The log-likelihood function for a random sample $\boldsymbol{x}=\left(x_{1}, \ldots, x_{n}\right)$ from (4) is written as:

$$
\begin{aligned}
\ell(\boldsymbol{\theta} \mid x) & =n \log B(\alpha, \beta)+(1-\alpha) \sum_{i=1}^{n} \log \left[\oint_{x_{i}}(\alpha, \beta)\right] \\
& +(1-\beta) \sum_{i=1}^{n} \log \left[1-\oint_{x_{i}}(\alpha, \beta)\right]
\end{aligned}
$$

The maximum likelihood estimates $\hat{\alpha}_{M L E}$ and $\hat{\beta}_{M L E}$ of $\alpha$ and $\beta$, respectively, are obtained numerically by maximizing, with respect to $\alpha$ and $\beta$, the log-likelihood function (8). Although it is necessary to solve a system of nonlinear equations to obtain $\hat{\alpha}_{M L E}$ and $\hat{\beta}_{M L E}$, this method has some advantages over the $L$-moments method. For example, using asymptotic arguments, it is trivial to estimate functions of $\alpha$ and $\beta$ and their standard errors as well as to build confidence intervals (Edwards, 1992). As pointed out by Glass (2000), Hosking (1990) obtained an asymptotic result specifying the sample $L$-moments have a multivariate normal distribution as $n \rightarrow \infty$. However, the standard errors depends on the distribution of the variable. So in order to be able to build confidence intervals it would be necessary to know the distribution of the variable. 


\section{Simulation results}

In this section the results are showed from a Monte Carlo experiment designed to investigate the sampling properties of the aforementioned estimation methods and identify those with the lowest bias and root mean-squared error for small to large sized samples drawn from the CB distribution. Sample sizes have been taking ranging from 10 to 100 by 10 and parameter values $(\alpha, \beta)=(0.2,0.2),(0.2,1.5),(0.5,0.2),(0.5,0.5),(1.0,0.2)$ and $(1.5$, 1.5) leading to all possible shapes of (4), showed in Figure 1. For each combination of $n, \alpha$ and $\beta M=10,000$ pseudo-random samples from the CB distribution have been generated using the inverse transform method. The relative bias (RBias) and relative root mean-squared error (RRMSE) were calculated as follows:

$$
\text { RBias }=\frac{1}{M} \sum_{j=1}^{M}\left(\frac{\hat{\theta}_{j}-\theta_{j}}{\theta_{j}}\right) \quad \text { and } \quad \text { RRMSE }=\sqrt{\frac{1}{M} \sum_{j=1}^{M}\left(\frac{\hat{\theta}_{j}-\theta_{j}}{\theta_{j}}\right)^{2}}
$$

where $\boldsymbol{\theta}_{\boldsymbol{j}}$ and $\widehat{\boldsymbol{\theta}}_{\boldsymbol{j}}$ are the $j$ th coordinates of $\boldsymbol{\theta}=(\alpha, \beta)$ and $\widehat{\boldsymbol{\theta}}=\left(\widehat{\alpha}_{M L E}, \widehat{\beta}_{M L E}\right)$ or $\widehat{\boldsymbol{\theta}}=$ $\left(\widehat{\alpha}_{L M E}, \widehat{\beta}_{L M E}\right)$.

All simulations were performed in Ox Console (Doornik, 2007), using the MaxBFGS function to obtain the maximum likelihood estimates of $\alpha$ and $\beta$, while for the $L$-moments method the estimates were obtained analytically from the equations presented in (7).

Plots of the estimated relative bias and estimated RRMSE versus $n$, for all parameters combinations, are showed in Figures 3 and 4, respectively. Figure 3 shows smaller estimated relative bias of $\hat{\alpha}_{M L E}$ and $\hat{\beta}_{M L E}$ as compared with the estimated relative bias of $\hat{\alpha}_{L M E}$ and $\hat{\beta}_{L M E}$, mainly for small and moderate sample sizes. In these plots, it was also observed that MLEs are negatively biased and LMEs are positively biased, moreover, both methods are returning asymptotically unbiased estimates. However, in the maximum likelihood method the estimated bias of $\alpha$ varies from -0.042 to -0.006 whereas in the $L$-moments method the variation is from 0.004 to 0.238 . Similar variations are observed for $\beta$.

Figure 4 shows that the estimated RRMSE for $\hat{\alpha}_{M L E}$ and $\hat{\beta}_{M L E}$ are smaller than $\hat{\alpha}_{L M E}$ and $\hat{\beta}_{L M E}$, especially for small sample sizes. The estimated RRMSE for $\hat{\alpha}_{M L E}$ varies from 0.100 to 0.344 whereas for $\hat{\alpha}_{L M E}$ the variation is from 0.101 to 0.641 . Similar variations are observed for $\hat{\beta}_{M L E}$ and $\hat{\beta}_{L M E}$.

Also, Figures 3 and 4 are showing that the estimated bias and estimated RRMSE are less sensitive to the values of $\alpha$ and $\beta$ in the MLE method. 
[Figure 3 about here.]

[Figure 4 about here.]

Finally, the overall performance was assessed of each estimation method, regardless of the parameters values, through two measures introduced in Cribari-Neto and Vasconcellos (2002). The authors called these measures as integrated bias squared (IBSQ) and average root mean-squared error (ARNSE). They are defined as follows:

$$
\operatorname{IBSQ}_{(k)}=\sqrt{\frac{1}{6} \sum_{h=1}^{6}\left(r_{h, k}\right)^{2}} \quad \text { and } \quad \operatorname{ARMSE}_{(k)}=\frac{1}{6} \sum_{h=1}^{6} R M S E_{h, k}
$$

where $r_{h, k}$ and $R M S E_{h, k}$ are the biases and the root mean-squared errors. These quantities are calculated for scenario $k, k=1, \ldots, 6$ and the results are showed in Figure 5. It is observed that the integrated bias squared and average root mean-squared error are smaller in the MLE, corroborating with the results from Figures 3 and 4.

[Figure 5 about here.]

\section{Applications}

In this section, the performance of the two estimation methods will be compared, considering four data sets on climate indices based on time series of temperature. The indices are TN10p, TN90p, TX10p and TX90p. From Dashkhuu et al. (2015), TN10p is an index measuring the percentage of time with daily minimum temperatures lower than the 10th percentile of minimum temperatures calculated for each calendar day (with reference to the climatological norm) using a running 5-day window. This is a measure of the percentage of unseasonably low-temperature nights (cool nights) in a year. Similarly, TX10p is an index showing the percentage of unseasonably low-temperature days (cool days). TN90p and TX90p are indices corresponding to the percentage of unseasonably high-temperature nights (warm nights) and days (warm days) in a year, respectively. Various additional details related to these and various other indexes can be found, for example, in Donat et al. (2013).

According to (Zhang et al., 2011) such indices allow straightforward monitoring of trends in the frequency or intensity of events, which can cause stress to humans or the environment. In this view, a probability distribution can be used for characterizing the indices behavior. Furthermore, it is important to consider a probability distribution that represents the nature of random variable, then the $\mathrm{CB}$ distribution it is a good choice, since it is flexible and its domain is in the unit interval. 
The indices from Rocha station in Uruguay available in ETCCDI (http://etccdi.pacificclimate.org/index.shtml) have been considered and the historical series from 1950 to 2000 . To the best of our knowledge, these indices have not been previously analyzed in the literature. Some descriptive measures for these indices are presented in Table 1.

[Table 1 about here.]

In Table 2 the parameter estimates and the corresponding standard errors are reported, under the maximum likelihood and $L$-moments methods, for the temperature extremes indices. It is possible to observe that the estimates are quite similar, except for TX90p indice. It is also interesting to note that the MLEs provides lower standard errors than the LME, which means more accurate estimates than LME.

[Table 2 about here.]

In order to test whether the data sets fits the CB distribution, three goodness-of-fit tests have been performed based on Kolmogorov-Smirnov (KS), Cramér-von-Mises (CvM) and Anderson-Darling (AD) statistics. The results are given in Table 3.From these table it is possible to conclude that $\mathrm{CB}$ distribution provides a good fit to these data sets and the estimation methods are quite similar for TN10p, TN90p and TX10p indices, while for the TX90p indice the MLE provide better fit than the LME. These conclusion are also supported by Figure 6, where we plot the empirical versus fitted CDF.

[Table 3 about here.]

[Figure 6 about here.]

Finally, to assess and compare the fits by the two estimation methods the concordance correlation coefficient (CCC) due to Lin (1989) was used. CCC is used to evaluate the agreement between the empirical and theoretical probabilities by measuring the variation of their linear relationship from the $45^{\circ}$ line through the origin (Lin, 1992). It is estimated as follows:

$$
\hat{\rho}_{c}=\frac{2 \hat{\sigma}_{12}}{\hat{\sigma}_{1}^{2}+\hat{\sigma}_{2}^{2}+\left(\hat{\mu}_{1}-\hat{\mu}_{2}\right)^{2}}
$$

where $\widehat{\mu}_{1}$ and $\widehat{\sigma}_{1}^{2}$ denote the sample mean and variance of empirical probabilities, $\hat{\mu}_{2}$ and $\hat{\sigma}_{2}^{2}$ denote the sample mean and variance of theoretical probabilities, and $\hat{\sigma}_{12}$ is the covariance between the empirical and theoretical probabilities. 
In Table 4 is presented, for MLE and LME the estimated CCC $\left(\hat{\rho}_{c}\right)$ between the empirical and theoretical probabilities, its Bootstrap bias-corrected version $\left(\tilde{\rho}_{c}\right)$, the $95 \%$ Bootstrap confidence interval and the descriptive scale suggested by McBride (2005). From this table it is evident that $\mathrm{CB}$ distribution provides a good fit to the indices, since the CCC values were higher than 0.98 . Finally, it is possible to see that LME has marginal largest values of $\mathrm{CCC}$, indicating a better estimate of parameter, hence the best fit to these indices.

[Table 4 about here.]

\section{Conclusions}

The CB distribution has been introduced by Jones (2002) and has not drawn attention for modeling data on the unit interval despite of the flexibility it provides in terms of the variety of shapes it can accommodate. In this paper it was compared, via intensive simulation, two methods of estimation of its parameters, namely the $L$-moments method and the maximum likelihood method. From our simulations it was discovered that the estimates are asymptotically unbiased and consistent, regardless of the estimation method. However, in small samples, MLE is the most appropriate method. Four applications considering temperature extremes were given to demonstrate that the $\mathrm{CB}$ distribution can be used quite effectively to provide good fits.

\section{Acknowledgments}

The authors would like to thank the Editor and the anonymous referee for their careful reading and for their comments which greatly improved the paper. 


\section{References}

[1] Cribari-Neto, F., Vasconcellos, K. L. P., 2002. Nearly unbiased maximum likelihood estimation for the Beta distribution. Journal of Statistical Computation and Simulation 72 (2), 107-118.

[2] Dashkhuu, D., Kim, J. P., Chun, J. A., Lee, W., 2015. Long-term trends in daily temperature extremes over Mongolia. Weather and Climate Extremes 8, 26-33.

[3] Dixon, A. C., 1902. Summation of a certain series. Proceedings of the London Mathematical Society s1-35 (1), 284-291.

[4] Donat, M. G., Alexander, L. V., Yang, H., Durre, I., Vose, R., Dunn, R. J. H., Willett, K. M., Aguilar, E., Brunet, M., Caesar, J., Hewitson, B., Jack, C., Klein Tank, A. M. G., Kruger,A. C., Marengo, J., Peterson, T. C., Renom, M., Oria Rojas, C., Rusticucci, M., Salinger, J., Elrayah, A. S., Sekele, S. S., Srivastava, A. K., Trewin, B., Villarroel, C., Vincent, L. A., Zhai, P., Zhang, X., Kitching, S., 2013. Updated analyses of temperature and precipitation extreme indices since the beginning of the twentieth century: The HadEX2 dataset. Journal of Geophysical Research: Atmospheres 118 (5), 2098-2118.

[5] Doornik, J. A., 2007. Object-Oriented Matrix Programming Using Ox, 3rd ed. London: Tim-berlake Consultants Press and Oxford.

[6] Edwards, A. W. F., 1992. Likelihood (Expanded Edition). Johns Hopkins University Press, Baltimore.

[7] Erişoğlu, Ü., Erişoğlu, M., 2014. L-Moments estimations for mixture of Weibull distributions. Journal of Data Science 12 (1), 69-85.

[8] Glass, S., 2000. Performance of bootstrap confidence intervals for L-moments and ratios of L -moments. Ph.D. thesis, School of Graduate Studies, East Tennessee State Universit, Electronic Theses and Dissertations. Paper 1. http://dc.etsu.edu/etd/1.

[9] Gradshteyn, I., Ryzhik, I., 1994. Table of Integrals, Series, and Products. Academic Press.

[10] Gupta, A. K., Nadarajah, S., 2004. Handbook of Beta Distribution and Its Applications. CRC Press. 
[11] Hosking, J. R. M., 1990. L-Moments: Analysis and estimation of distributions using linear combinations of order statistics. Journal of the Royal Statistical Society. Series B (Method-ological) 52 (1), 105-124.

[12] Hosking, J. R. M., Wallis, J. R., 1997. Regional Frequency Analysis: An Approach Based on L-Moments. Cambridge University Press.

[13] Iacobellis, V., 2008. Probabilistic model for the estimation of T year flow duration curves.Water Resources Research 44 (2), w02413.

[14] Johnson, N. L., Kotz, S., Balakrishnan, N., 1995. Continuous Univariate Distributions., 2nd Edition. Vol. 2. John Wiley \& Sons Inc., New York.

[15] Jones, M. C., 2002. The complementary Beta distribution. Journal of Statistical Planning and Inference 104 (2), 329-337.

[16] Jones, M. C., 2007. On a class of distributions defined by the relationship between their density and distribution functions. Communications in Statistics - Theory and Methods 36 (10), 18351843 .

[17] Lin, L. I., 1989. A concordance correlation coefficient to evaluate reproducibility. Biometrics 45 (1), 255-268.

[18] Lin, L. I., 1992. Assay validation using the concordance correlation coefficient. Biometrics 48 (2), 599-604.

[19] McBride, G., 2005. A proposal for strength-of-agreement criteria for Lin's concordance correlation coefficient. NIWA Client Report: HAM2005-062.

[20] Nadarajah, S., Kotz, S., 2007. Multitude of Beta distributions with applications. Statistic: A Journal of Theoretical and Applied Statistics 41 (2), 153-179.

[21] Zhang, X., Alexander, L., Hegerl, G. C., Jones, P., Tank, A. K., Peterson, T. C., Trewin, B., Zwiers, F. W., 2011. Indices for monitoring changes in extremes based on daily temperature and precipitation data. Wiley Interdisciplinary Reviews: Climate Change 2 (6), 851-870. 

DISTRIBUTION WITH APPLICATIONS ON TEMPERATURE EXTREMES

Table 1: Descriptive measures for TN10p, TN90p, TX10p and TX90p.

\begin{tabular}{cccccccccc}
\hline Indices & $n$ & Missing & Min & Mean & Median & Max & Std Dev & Skewness & Kurtosis \\
\hline TN10p & 38 & 13 & 0.0446 & 0.1008 & 0.0948 & 0.1519 & 0.0300 & 0.1161 & 1.8875 \\
TN90p & 38 & 13 & 0.0567 & 0.1026 & 0.0998 & 0.1650 & 0.0299 & 0.2908 & 2.1358 \\
TX10p & 35 & 16 & 0.0503 & 0.1077 & 0.1031 & 0.1773 & 0.0284 & 0.4404 & 2.7756 \\
TX90p & 35 & 16 & 0.0275 & 0.0987 & 0.1014 & 0.1764 & 0.0318 & 0.0268 & 3.0734 \\
\hline
\end{tabular}

Table 2: Parameter estimates (standard errors) for the annual temperature extremes indices.

\begin{tabular}{|c|c|c|c|c|c|c|c|c|}
\hline \multirow{3}{*}{$\begin{array}{l}\text { Indices } \\
\text { TN10p }\end{array}$} & \multicolumn{4}{|c|}{ MLE } & \multicolumn{4}{|c|}{ LME } \\
\hline & \multicolumn{2}{|c|}{$\hat{\alpha}$} & \multicolumn{2}{|c|}{$\hat{\beta}$} & \multicolumn{2}{|c|}{$\hat{\alpha}$} & \multicolumn{2}{|c|}{$\hat{\beta}$} \\
\hline & 0.2129 & $(0.0318)$ & 0.0239 & $(0.0037)$ & 0.2137 & $(0.0331)$ & 0.0239 & $(0.0039)$ \\
\hline TN90p & 0.2042 & $(0.0304)$ & 0.0234 & $(0.0036)$ & 0.2078 & $(0.0321)$ & 0.0238 & $(0.0038)$ \\
\hline TX10p & 0.1753 & $(0.0268)$ & 0.0211 & $(0.0034)$ & 0.1803 & $(0.0288)$ & 0.0218 & $(0.0036)$ \\
\hline TX90p & 0.2288 & $(0.0356)$ & 0.0254 & $(0.0042)$ & 0.2277 & $(0.0371)$ & 0.0249 & $(0.0043)$ \\
\hline
\end{tabular}

Table 3: One-sample Kolmogorov-Smirnov goodness-of-fit test, Cramer-von Mises goodness-offit test and Anderson-Darling goodness-of-fit test ( $p$-values).

\begin{tabular}{|c|c|c|c|c|c|c|c|c|c|c|c|c|}
\hline \multirow{3}{*}{$\frac{\text { Indices }}{\text { TN10p }}$} & \multicolumn{4}{|c|}{$\mathrm{KS}$} & \multicolumn{4}{|c|}{ CvM } & \multicolumn{4}{|c|}{$\mathrm{AD}$} \\
\hline & \multicolumn{2}{|c|}{ MLE } & \multicolumn{2}{|c|}{ LME } & \multicolumn{2}{|c|}{ MLE } & \multicolumn{2}{|c|}{ LME } & \multicolumn{2}{|c|}{ MLE } & \multicolumn{2}{|c|}{ LME } \\
\hline & 0.1217 & $(0.1021)$ & 0.1239 & $(0.1505)$ & 0.0846 & $(0.1140)$ & 0.0833 & $(0.1872)$ & 0.5416 & $(0.1258)$ & 0.5408 & $(0.1598)$ \\
\hline TN90p & 0.1052 & $(0.2465)$ & 0.1040 & $(0.3516)$ & 0.0555 & $(0.3361)$ & 0.0506 & $(0.4882)$ & 0.3555 & $(0.3981)$ & 0.3333 & $(0.4874)$ \\
\hline TX10p & 0.0894 & $(0.5844)$ & 0.0815 & $(0.8027)$ & 0.0326 & $(0.7339)$ & 0.0319 & $(0.8083)$ & 0.2225 & $(0.7999)$ & 0.2132 & $(0.8357)$ \\
\hline TX90p & 0.1149 & $(0.2030)$ & 0.1286 & $(0.1486)$ & 0.0689 & $(0.2121)$ & 0.0855 & $(0.1696)$ & 0.4356 & $(0.2499)$ & 0.4825 & $(0.2206)$ \\
\hline
\end{tabular}

Table 4: Concordance correlation coefficient between empirical and theoretical probabilities.

\begin{tabular}{ccccccc}
\hline Indices & Method & $\hat{\rho}_{c}$ & $\tilde{\rho}_{c}$ & LI & LS & Strength of agreement \\
\hline \multirow{2}{*}{ TN10p } & MLE & 0.9876 & 0.9884 & 0.9782 & 0.9932 & Substantial \\
& LME & 0.9878 & 0.9885 & 0.9783 & 0.9933 & Substantial \\
\hline \multirow{2}{*}{ TN90p } & MLE & 0.9920 & 0.9925 & 0.9858 & 0.9956 & Almost perfect \\
& LME & 0.9927 & 0.9931 & 0.9869 & 0.9959 & Almost perfect \\
\hline \multirow{2}{*}{ TX10p } & MLE & 0.9949 & 0.9952 & 0.9905 & 0.9974 & Almost perfect \\
& LME & 0.9950 & 0.9953 & 0.9907 & 0.9974 & Almost perfect \\
\hline \multirow{2}{*}{ TX90p } & MLE & 0.9885 & 0.9892 & 0.9792 & 0.9935 & Substantial \\
& LME & 0.9857 & 0.9868 & 0.9727 & 0.9923 & Substantial \\
\hline
\end{tabular}



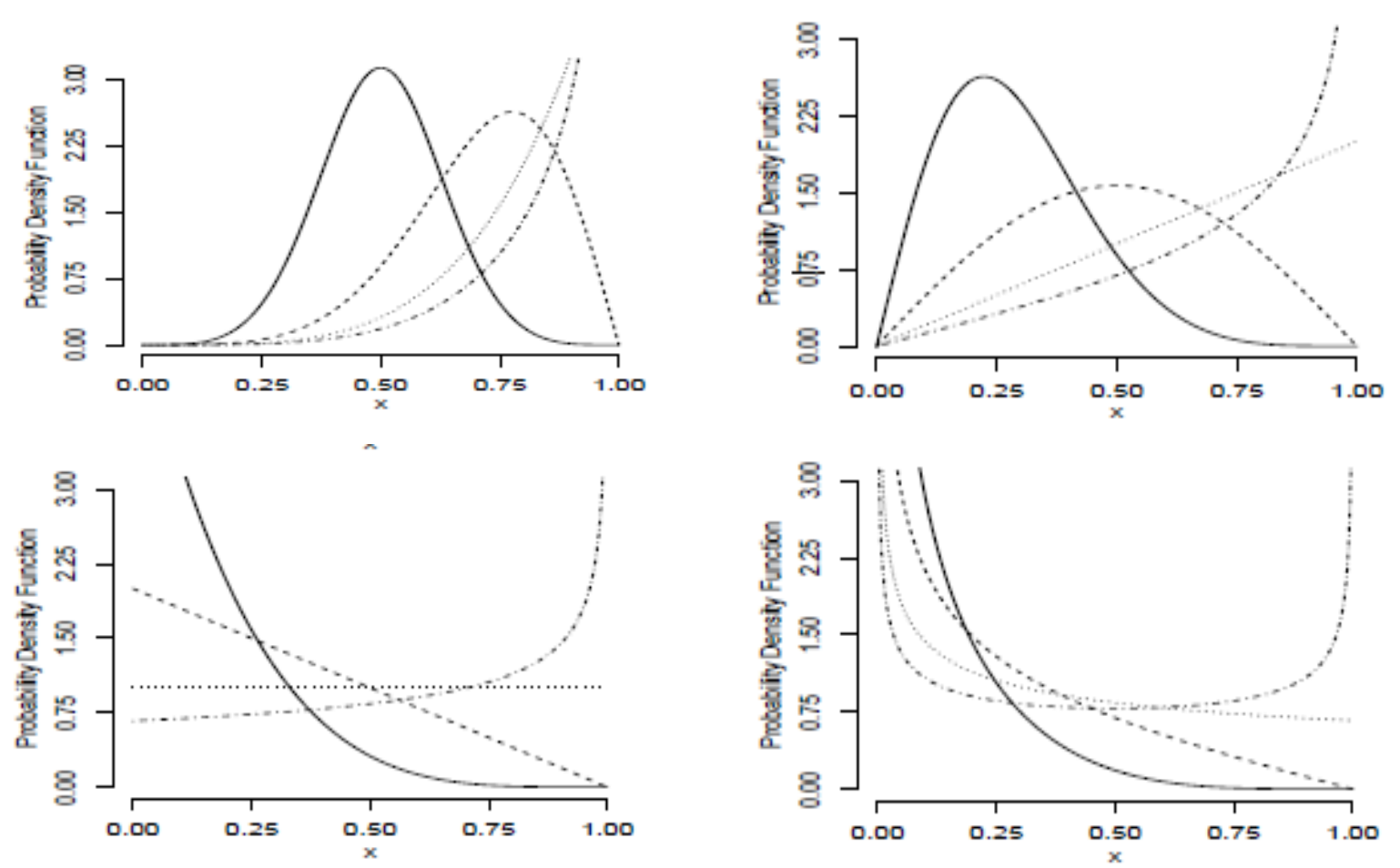

Figure 1: Probability density function of CB distribution for selected values of $\alpha$ and $\beta$. (left upper panel: $\alpha=0.2$, right upper panel: $\alpha=0.5$, left lower panel: $\alpha=1.0$ and right lower panel: $\alpha=1.5$; solid line: $\beta=0.2$, dashed line: $\beta=0.5$, dotted line: $\beta=1.0$ and dotdash line: $\beta=$ 1.5).
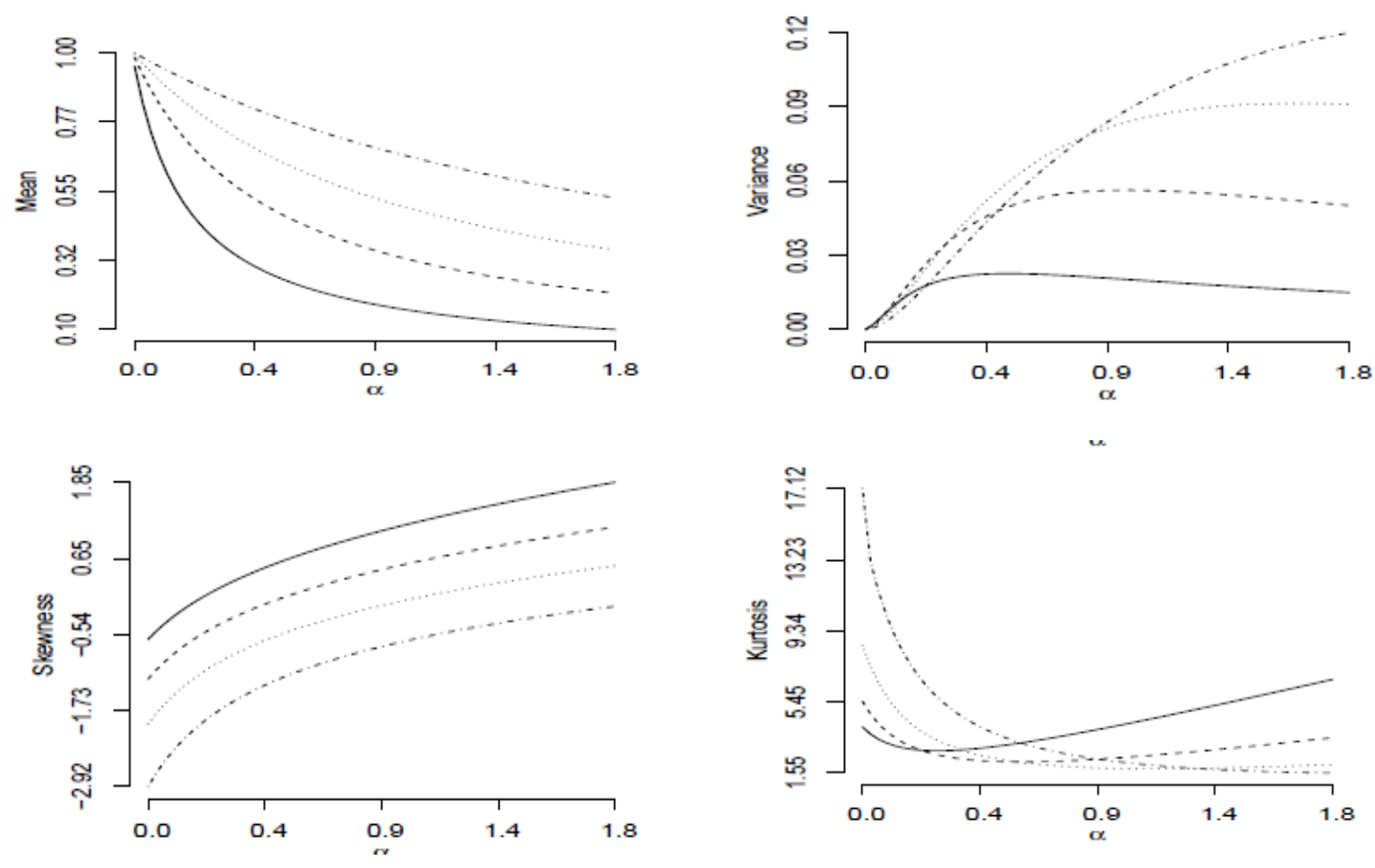

Figure 2: Behavior of the mean, variance, skewness and kurtosis of $\mathrm{CB}$ distribution as a function of $\alpha$. (solid line: $\beta=0.2$, dashed line: $\beta=0.5$, dotted line: $\beta=1.0$ and dotdash line: $) \beta=2.0$ 

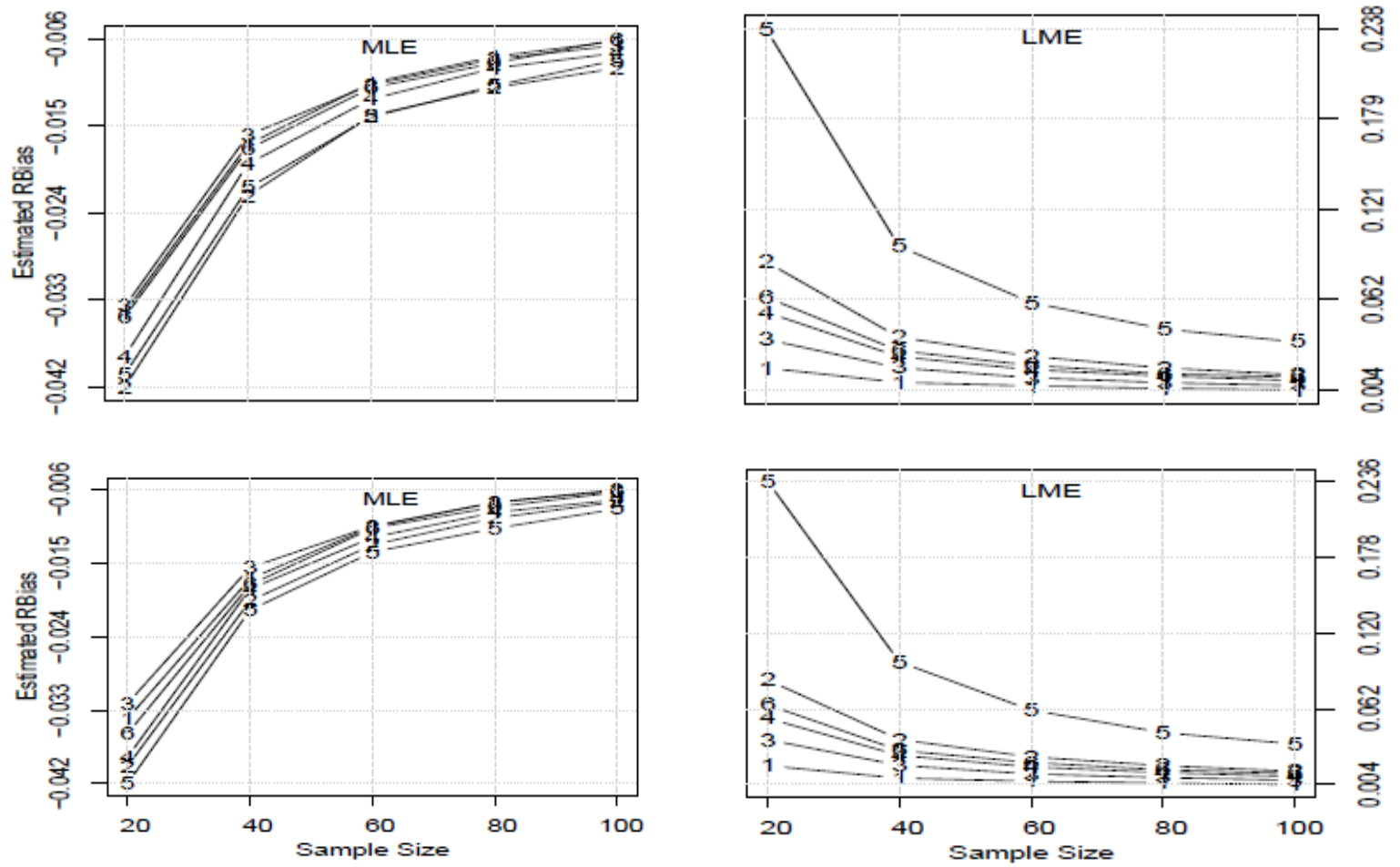

Figure 3: Upper Panel: estimated relative bias of $\alpha$. Lower Panel: estimated relative bias of $\beta$. 1: $(\alpha, \beta)=(0.2,0.2), 2:(\alpha, \beta)=(0.2,1.5), 3:(\alpha, \beta)=(0.5,0.2), 4:(\alpha, \beta)=$ $(0.5,0.5), 5:(\alpha, \beta)=(1.0,0.2)$ and $6:(\alpha, \beta)=(1.5,1.5)$. 

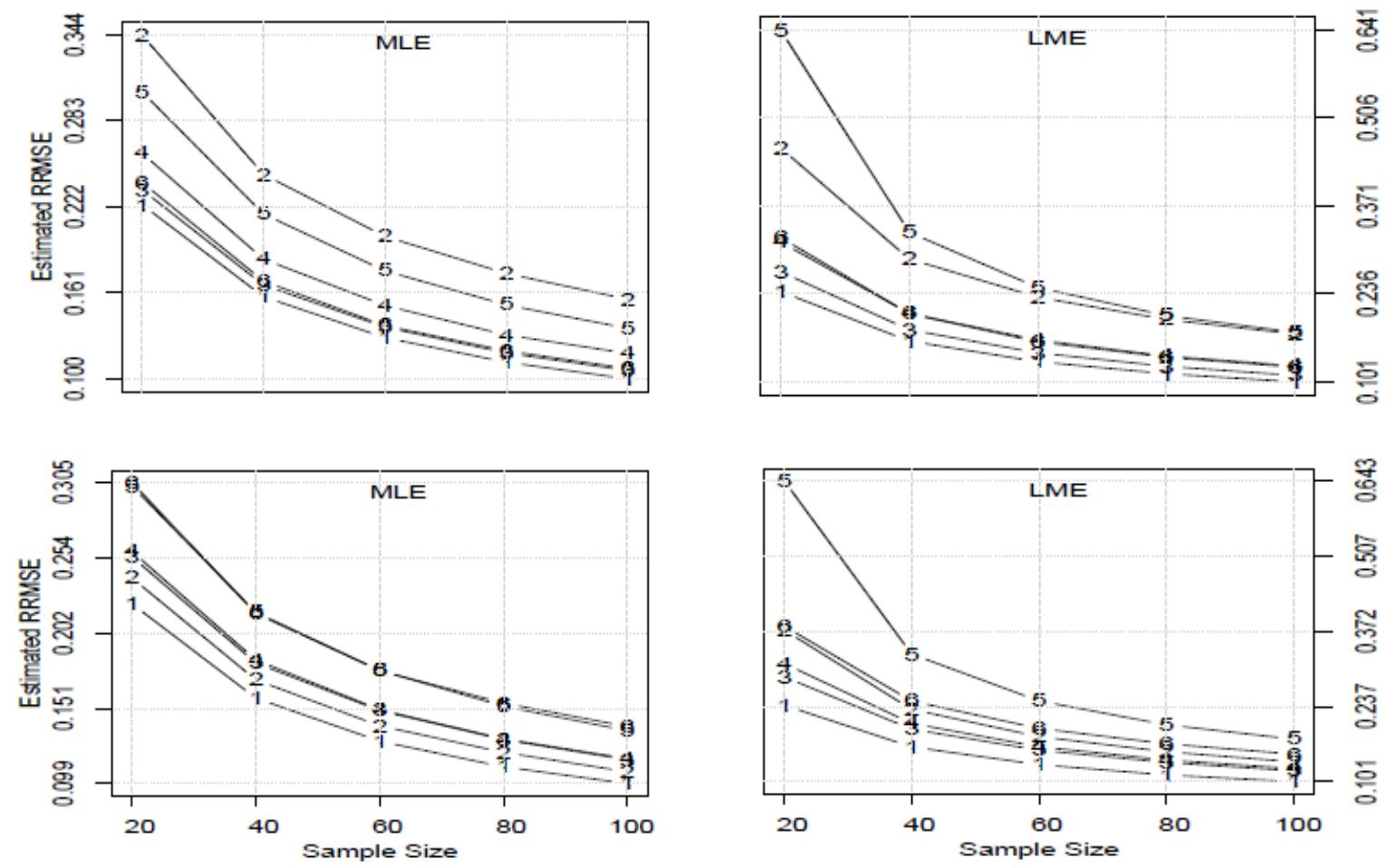

Figure 4: Upper Panel: estimated relative root mean-squared error of $\alpha$. Lower Panel: estimated relative root mean-squared error of $\beta .1:(\alpha, \beta)=(0.2,0.2), 2:(\alpha, \beta)=(0.2,1.5), 3:(\alpha, \beta)=$ $(0.5,0.2), 4:(\alpha, \beta)=(0.5,0.5), 5:(\alpha, \beta)=(1.0,0.2)$ and $6:(\alpha, \beta)=(1.5,1.5)$.
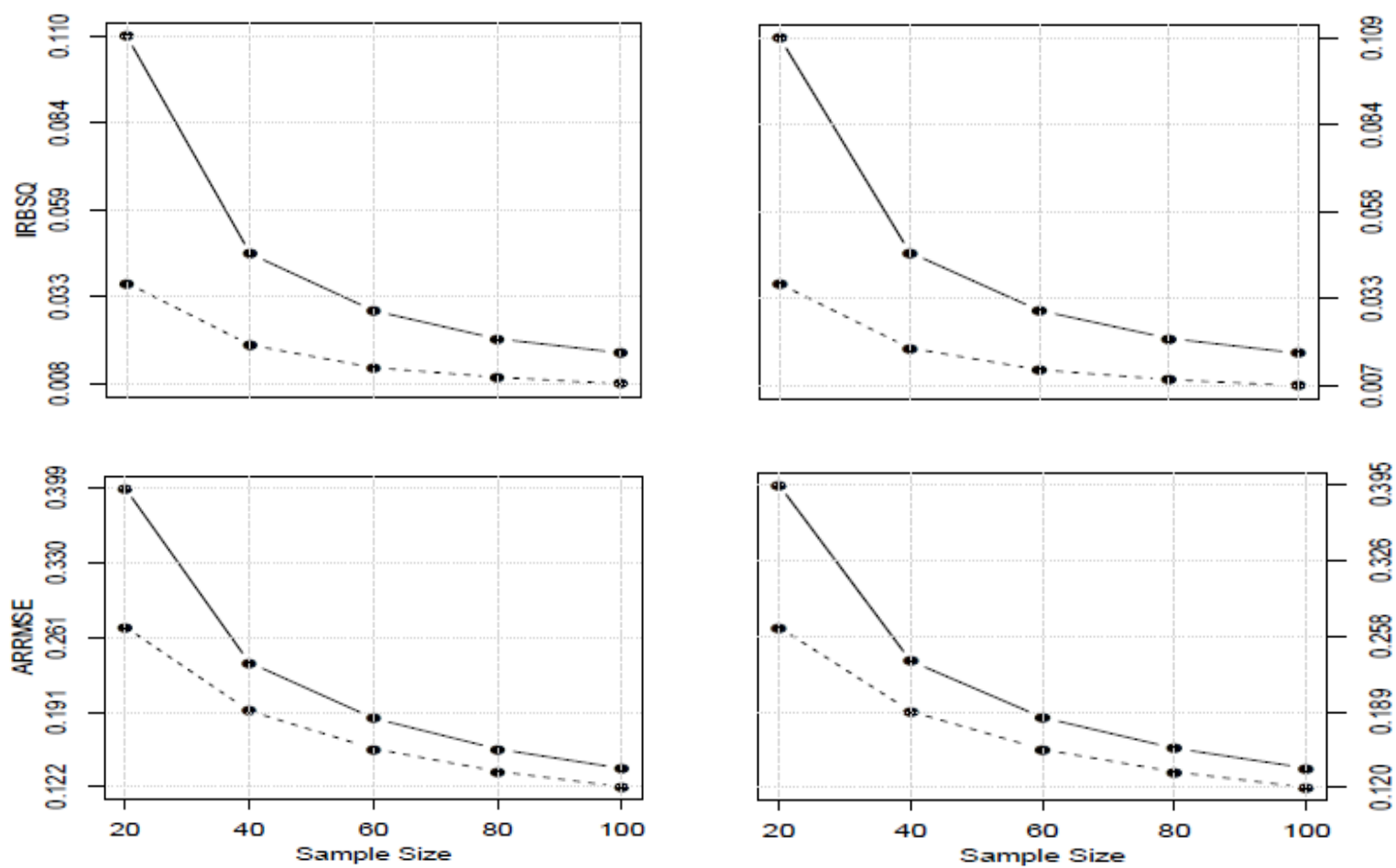

Figure 5: Left Upper Panel: IRBSQ of $\alpha$. Right Upper Panel: IRBSQ of $\beta$. Left Lower Panel: ARRMSE of $\alpha$. Right Lower Panel: ARRMSE of $\beta$. solid line: LME and dashed line: MLE. 

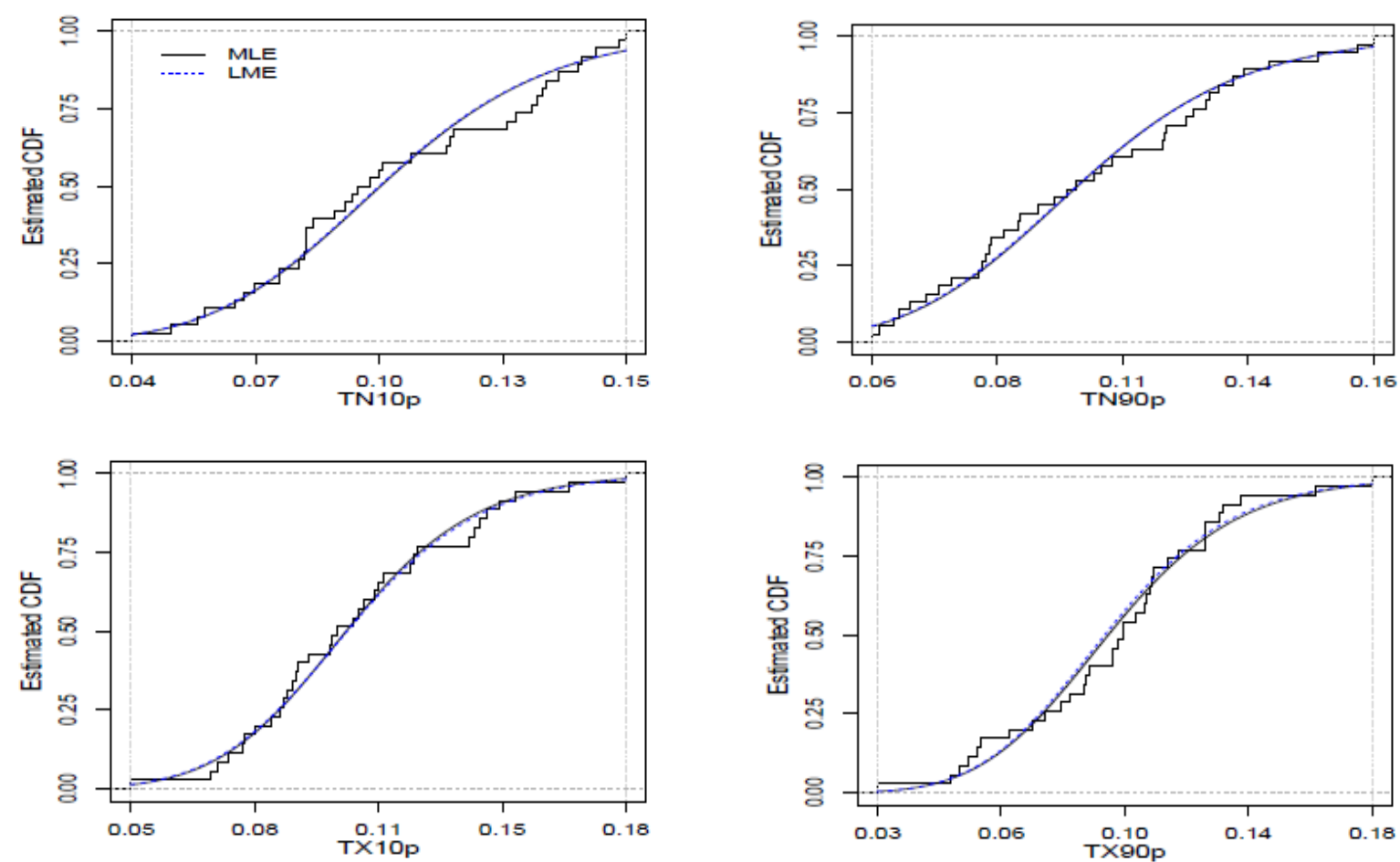

Figure 6: Empirical and fitted CDF for the annual temperature extremes indices. 